\title{
Modeling the Magnetic-Hyperthermia Response of Linear Chains of Nanoparticles with Low Anisotropy: A Key to Improving Specific Power Absorption
}

\author{
Daniela P. Valdés $\odot,{ }^{1,2}$ Enio Lima Jr., ${ }^{1}$ Roberto D. Zysler $\odot,{ }^{1,2}$ and Emilio De Biasi® ${ }^{1,2, *}$ \\ ${ }^{1}$ Instituto de Nanociencia y Nanotecnología, CNEA-CONICET, R8402AGP San Carlos de Bariloche, \\ Río Negro, Argentina \\ ${ }^{2}$ Instituto Balseiro, Universidad Nacional de Cuyo, R8402AGP San Carlos de Bariloche, Río Negro, Argentina
}

(Received 6 April 2020; revised 14 May 2020; accepted 4 June 2020; published 9 July 2020)

The effect of magnetic interactions is a key issue for the performance of nanoparticles in magnetic fluid hyperthermia. There are reports informing on beneficial or detrimental effects in terms of the specific power absorption depending on the intrinsic magnetic properties and the spatial arrangement of the nanoparticles. To understand this effect, our model treats a simple system: an ensemble of identical nanoparticles arranged in an ideal chain with the easy axis of the effective uniaxial anisotropy of each particle aligned parallel to the chain. We study the magnetic relaxation of linear chains with low anisotropy in magnetic-fluid-hyperthermia experiments, a system that yields a larger hysteresis area than the noninteracting case (i.e., improved specific power absorption) for all orientations of the chain (even in the perpendicular configuration and the randomly oriented case). The most-favorable case is the chain parallel to the external field; however, we show that the incorporation of a dipolar-field component perpendicular to the external field is necessary for the correct modeling of chains nearly in the perpendicular configuration, which is not always done. The mechanism involved in the hysteresis-area increase can be interpreted as a shift between the local field and the applied field.

DOI: 10.1103/PhysRevApplied.14.014023

\section{INTRODUCTION}

Magnetic fluid hyperthermia (MFH) consists in the controlled local heating of tissue through the magnetic losses of magnetic nanoparticles (NPs) in the presence of an ac magnetic field [1-3]. MFH is based on the fact that increasing the temperature to $41-42^{\circ} \mathrm{C}$ can preferably kill tumor cells rather than normal ones [4]. When NPs are exposed to an ac magnetic field, they absorb energy from the field, subsequently converting it into heat. The energy absorbed by the mass of NPs per unit time is called "specific power absorption" (SPA) and can be obtained from the area enclosed by a magnetization hysteresis loop times the frequency of the experiment $f$. The hysteresis loop arises from the dephasing between the applied ac field and the response of the magnetic moment of the material [5]. The response of the magnetic moment of the NPs is directly related to the magnetic properties of the system.

For applications in MFH, the size and controlled fabrication of magnetic NPs are crucial issues to obtain the desired magnetic properties. The advances achieved nowadays allow the fabrication of NPs with detailed control of their size and composition [6,7]. However, there are many

\footnotetext{
*debiasi@cab.cnea.gov.ar
}

aspects associated with the characteristics of the NPs that are relevant to their performance; for instance, interfacial effects in core-shell systems, anisotropy, and interparticle interactions [8-10].

The evaluation of interactions and their effect on hysteresis loops is crucial for the success of MFH experiments due to NP aggregation promoted in intracellular environments [11]. Interparticle interaction is inherent to such experiments because aggregation is caused not only by the cellular environment but also by other factors, such as the applied field gradient [12] or even the NP synthesis procedure [13].

Many efforts are being made to assess the influence of aggregation on the magnetic response of NPs, from both the experimental and the theoretical point of view [1416]; however, it is a complex problem. The varied nature of NP agglomerates with distinct spatial arrangements (clusters [16,17], nanoflowers [18], rings [19], chains [20-24], natural magnetosomes synthesized by magnetotactic bacteria [25], etc.) and the large set of experimental conditions make the task of determining the effect of interactions on the SPA of the system extremely challenging. In addition, the orientation of the external applied magnetic field with respect to these arrangements is also decisive for the results of the experiments [24]. 
Chainlike assemblies have shown great performance in MFH [20-24]. The Néel relaxation time in systems with similar characteristics is affected by interparticle dipolar interactions, as evidenced by the work of Anand et al. [26]. However, there is some controversy regarding this subject, as some authors have reported opposing results for other linear systems, such as Zubarev and Abu-Bakr [27,28]. Moreover, there are some reports on NP aggregates with different anisotropy giving different properties [29].

From the aforementioned discussion, ideal NP-chain arrangements are good systems for the study and characterization of the fundamental effects of dipolar interactions on the magnetic response under an ac magnetic field. In this paper, we study in detail the mechanisms involving dipolar interactions in the magnetic relaxation of an ideal system for MFH experiments. Our system is built up in a simple way to gather and understand the essential effects caused by interactions in these experiments. We consider an ideal chain of low-anisotropy NPs with their easy axes of effective uniaxial anisotropy in the direction of the chain, allowing different orientations of the chain with respect to the applied field. The low-anisotropy assumption means that the anisotropy field $H_{K}$ is lower than the amplitude of the ac applied field $H_{0}$. For this system, $H_{K}<H_{0}$ and interactions are considered; consequently the linear response theory [30] cannot be applied. Hence, another nonlinear model $[13,31]$ is used for this study.

This paper is organized in four sections: Sec. I provides an introduction, Sec. II introduces the model used and details its hypotheses, in Sec. III we show and discuss the results of our numerical simulations, and Sec. IV presents the conclusion of this work.

\section{THE MODEL}

The model used in this work is based on a previous one, in which the magnetic behavior of an ideal assembly of noninteracting NPs is described as a function of the external magnetic field and temperature [31]. We have successfully used this model in various situations: to study the effect of thermal fluctuations on the ferromagnetic resonance of superparamagnetic particle and blocked-particle systems [32] and to characterize the precision of firstorder-reversal-curve analysis for NP systems [33]. The model was also effectively used to describe MFH experiments; for example, to determine the influence of interparticle interactions in simple agglomerates dispersed in toluene [13] and on the intracellular medium [34]. The central idea is based on the fact that temperature plays two fundamental roles: on the one hand, it helps to invert the NP magnetic moment from one minimum to the other and, on the other hand, it is responsible for reducing the effective value of magnetization due to thermal fluctuations [31]. The fraction of NPs that invert their magnetic moment is given by the probability of finding a particle in the superparamagnetic regime $L=1-\exp \left(-\tau_{m} / \tau\right)$ [13,31-33], where $\tau_{m}$ is the measurement time and $\tau$ is the effective relaxation time. The effects of fluctuations on each energy minimum are calculated by the statistical averaging on the regions of the corresponding minimum $[35,36]$.

\section{A. Noninteracting case}

We assume a noninteracting single-domain NP system with uniaxial anisotropy whose magnetic relaxation is given by the reversal of the magnetic moment (i.e., Néel relaxation). Our noninteracting model is based on the Stoner-Wohlfarth model [35], in which the energy $E$ of a $\mathrm{NP}$, under the previous considerations, can be expressed as

$$
E=-\mu_{0} \boldsymbol{\mu} \cdot \mathbf{H}-K V(\hat{\mathbf{n}} \cdot \hat{\boldsymbol{\mu}})^{2},
$$

where $\mu_{0}$ is the vacuum permeability, $\boldsymbol{\mu}$ is the magnetic moment of the particle, $\mathbf{H}$ is the magnetic field, $K$ is its anisotropy constant, and $V$ is its volume. $\hat{\mathbf{n}}$ denotes the direction of the anisotropy easy axis. This energy expression yields two minima in which the NPs can fluctuate.

Although we talk about a single NP, in our study each of them represents an ensemble of NPs. The fraction of NPs in the ensemble on each energy minimum is $P_{k}, k=0,1$, and is called "population of the energy minimum $k$ " from this point forward. They satisfy $P_{0}+P_{1}=1$ and are time dependent because there is a probability $L$ that a NP will fluctuate between minima (i.e., the probability of being in the superparamagnetic regime). Given $P_{0}(t)$ and $P_{1}(t)$, the populations at time $t$, the evolution of the population of the minima in a discritized time interval $\delta t$ corresponds to

$$
P_{0}(t+\delta t)=P_{0}(t)+L\left[P_{0}^{\infty}-P_{0}(t)\right],
$$

where $P_{0}^{\infty}$ is the equilibrium population associated with the energy minimum 0 . In this way, if $\delta H$ is the field step, then $\delta H / \delta t$ is the field sweep speed, which can be calculated in a MFH experiment as $2 H_{0} f$. Equation (2) can be derived through the master equation and the Arrhenius law [31], giving the closed-form expression $L=L\left(H, T, \tau_{m}\right)=$ $1-\exp \left(-\tau_{m} / \tau\right)$, with $T$ the temperature. The probability expression for $L$ is identical to that used in kinetic Monte Carlo (MC) simulations [26,36,37]. One difference between the two procedures is that in the kinetic MC simulations the statistical average is calculated in the usual way for MC simulations but in our model we perform numerical integrations weighted by the Boltzmann probability on the regions of interest.

As our model is probabilistic, to calculate the average magnetization of the system $\langle\mu\rangle=\langle\boldsymbol{\mu} \cdot \mathbf{H}\rangle$ we need to consider the superparamagnetic and the blocked contributions in the ensemble with probabilities $L$ and $1-L$, respectively. The fraction of NPs in the ensemble that are 


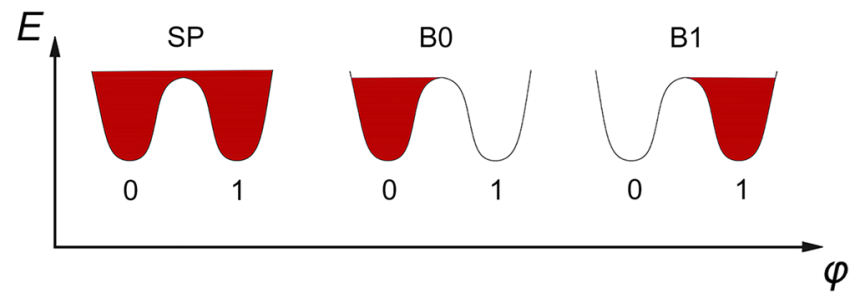

FIG. 1. The energy minima. The integration regions to obtain the different contributions to the statistical average of the magnetic moment are colored. $\mathrm{B} k$, blocked in minimum $k=0,1$; SP, superparamagnetic.

in the superparamagnetic regime are in thermodynamic equilibrium and their magnetization is a state function of $H$ and $T$. Meanwhile, the blocked NPs are not in thermodynamic equilibrium and their magnetization depends on their magnetic history and therefore they contribute to the hysteresis of the system. The average for the superparamagnetic fraction $\langle\mu\rangle_{\mathrm{SP}}=\langle\boldsymbol{\mu} \cdot \mathbf{H}\rangle_{\mathrm{SP}}$ is obtained by integration over all the microstates of the system. On the other hand, for the blocked fraction, the contributions of each energy minimum $P_{k}\langle\mu\rangle_{B k}=P_{k}\langle\boldsymbol{\mu} \cdot \mathbf{H}\rangle_{B k}$ (with $k=$ $0,1)$ have to be taken into account separately by integrating wherever it corresponds. In Fig. 1, these integrations are schematized.

With all of these considerations in mind, the total average magnetization of the system $\langle\mu\rangle$ as a function of $t$ can be written as

$$
\langle\mu\rangle=L\langle\mu\rangle_{\mathrm{SP}}+(1-L) \sum_{k=0,1} P_{k}\langle\mu\rangle_{B k},
$$

where the time dependence is given through the evolution of the populations of the minima and the changes in the minima due to the external field.

\section{B. Including dipolar interactions}

In general, interparticle interactions are a complex problem to solve, and even more so for dipolar interactions, which are strongly dependent on the magnetic assembly of the system. As already mentioned, the nonlinear model has been modified to treat interacting NP systems before $[13,34]$. However, the previous approaches were for clusters (in particular slightly distorted hexagonal-closepacked arrangements) in the mean-field approximation. By contrast, in the present work we treat linear arrangements in the dipole-dipole approximation by calculating the dipolar field acting on each NP.

We consider systems of diluted chains of particles with uniaxial anisotropy. This means that the agglomeration level in our simulations is rather low. Moreover, collective behavior is not exhibited, as is expected for highly compact agglomerates of NPs with different anisotropy values
[38-40], contrarily to the case we are dealing with. For this reason, each NP "retains" its identity; that is, its magnetic state can be well described by the orientation of the magnetic moment of the particle. We assume that the NPs are not in contact, and thus no exchange is present and their interactions are only of dipolar nature.

The main idea is to include the dipolar interactions acting on each particle through a correction to its local field $\mathbf{H}_{\text {loc }}$. Hence, the effective magnetic field that governs the behavior of particle $i$ can be described by the external magnetic field $\mathbf{H}$ plus an interaction field $\mathbf{H}_{\mathrm{dip} i}$. Strictly, we treat each particle as noninteracting but with a different local field that accounts for the dipolar field produced by other NPs in its position in the chain. We can do this because we are dealing with diluted chain systems with mild to moderate interactions where collective behavior is not exhibited.

Several parameters are important when in a magnetic relaxation study: anisotropy, particle size, different kinds of agglomerates and spatial arrangements, etc. Another path to solve the problem is to set distributions of these parameters. Taking all of this into account, in this work, we focus on studying the most-relevant effects of dipolar interactions in one-dimensional chains of NPs, and for this we assume the particles to be identical, with the same size, anisotropy constant $K$, and saturation magnetization $M_{S}$, to avoid the impacts introduced by considering any distribution of these quantities that could mask the effects of interactions on the magnetic properties of the system.

Focusing on the hypotheses of our model, for this specific magnetic relaxation problem, we make the following assumptions:

(a) The magnetic relaxation of each NP can be individually described by the model presented in Sec. II A.

(b) A system of identical single-domain magnetic NPs with uniaxial anisotropy arranged in an equally spaced linear chain is considered.

(c) A diluted arrangement of chains is studied, which allows us to ignore the interaction between NPs from different chains.

(d) The particles positioned in the same chain interact dipolarly with each other.

(e) The easy axis of a given particle is aligned along the main direction of its corresponding chain. This is based on the idea that in a chain-formation process, the dipolar energy is minimized if the easy axes are all lined up in the chain direction.

(f) The Néel relaxation time $\tau$ for the NPs is much smaller than the time necessary to take $H$ from the maximum applied field $H_{0}$ to $-H_{0}$. That is, $f \tau \ll 1$, where $f$ is the frequency of the experiment. In addition, the step in which the field is swept is small enough to 
(a)

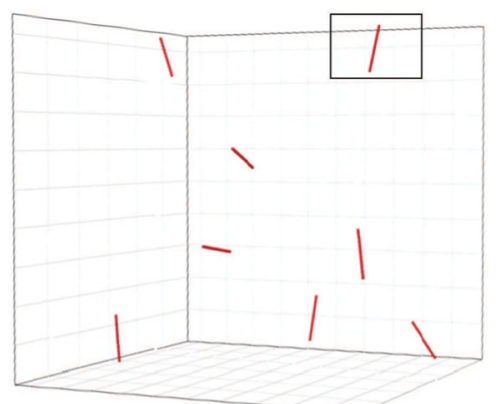

(b)

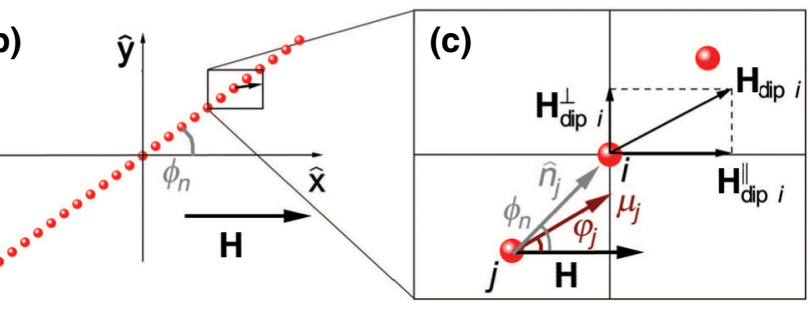

FIG. 2. (a) A system of diluted chains, (b) one of the chains with its orientation with respect to the external field, and (c) magnetic moment, anisotropy axis, and dipolar field (with each of its components) for individual particles of the chain.

ignore changes in the energy minima between time $t$ and time $t+\delta t$.

(g) We assume that $H_{K}=2 K / M_{S}<H_{0}$. In this way, we are sure that there are not minor loops in our simulations.

Under the aforementioned considerations, the energy of NP $i$ can be described by the Stoner-Wohlfarth model [35]. However, as we are dealing with an interacting system, the effective field on this particle is $\mathbf{H}+\mathbf{H}_{\text {dip } i}$ :

$$
E_{i}=-\mu_{0} \boldsymbol{\mu}_{i} \cdot\left(\mathbf{H}+\mathbf{H}_{\mathrm{dip} i}\right)-K_{i} V_{i}\left(\hat{\mathbf{n}}_{i} \cdot \hat{\boldsymbol{\mu}}_{i}\right)^{2},
$$

where $\mathbf{H}_{\mathrm{dip} i}=\sum_{j \neq i}\left\{-\boldsymbol{\mu}_{j} / r_{i j}^{3}+3\left[\mathbf{r}_{i j}\left(\boldsymbol{\mu}_{j} \cdot \mathbf{r}_{i j}\right) / r_{i j}^{5}\right]\right\}$ is the dipolar field acting over the magnetic moment of the $i$ th particle $\boldsymbol{\mu}_{i}, K_{i}$ is its anisotropy constant, $V_{i}$ is the particle's volume, $\hat{\mathbf{n}}_{i}$ is the direction of the easy axis, and $\mathbf{r}_{i j}$ is the vector that points from particle $j$ to $i$.

Under hypotheses (c)-(e), the equilibrium moment of a given particle, and as a consequence, the dipolar effective field on a particle, lies in the plane defined by the orientation of the anisotropy $\hat{\mathbf{n}}_{i}$ and the external magnetic field $\mathbf{H}=H \hat{\mathbf{x}}$. The system is illustrated in Fig. 2.

In this context, the dipolar field $\mathbf{H}_{\mathrm{dip} i}$ of a given particle can be decomposed in the parallel and perpendicular directions with respect to $\mathbf{H}$ as

$$
\begin{aligned}
& \mathbf{H}_{\mathrm{dip} i}^{\|}=\sum_{j \neq i} \frac{\left\langle\mu_{j}^{\|}\right\rangle}{D^{3}|j-i|^{3}}\left[3 \cos \left(\phi_{n}-\varphi_{j}\right) \cos \phi_{n}-\cos \varphi_{j}\right] \hat{\mathbf{x}}, \\
& \mathbf{H}_{\mathrm{dip} i}^{\perp}=\sum_{j \neq i} \frac{\left\langle\mu_{j}^{\perp}\right\rangle}{D^{3}|j-i|^{3}}\left[3 \cos \left(\phi_{n}-\varphi_{j}\right) \sin \phi_{n}-\sin \varphi_{j}\right] \hat{\mathbf{y}},
\end{aligned}
$$

respectively, where $D$ is the distance between the centers of adjacent NPs in the chain, and $\phi_{n}$ and $\varphi_{j}$ indicate the orientation of the chain and the magnetization with respect to $\mathbf{H}$, respectively (see Fig. 2). As $\mu_{j}^{\|}=\mu_{j} \cdot \hat{\mathbf{x}}$ and $\mu_{j}^{\perp}=$ $\boldsymbol{\mu}_{j} \cdot \hat{\mathbf{y}},\left\langle\mu_{j}^{\|}\right\rangle$and $\left\langle\mu_{j}^{\perp}\right\rangle$ denote the statistical average of the parallel and perpendicular components of the $j$ th magnetic moment.

Analogously to the noninteracting case (see Sec. II A), to calculate these averages it is necessary to take into account the superparamagnetic and blocked contributions with probabilities $L_{j}$ and $1-L_{j}$, respectively. Again, the averages for the superparamagnetic fraction, $\left\langle\mu_{j}^{\|}\right\rangle_{\mathrm{SP}}$ and $\left\langle\mu_{j}^{\perp}\right\rangle_{\mathrm{SP}}$, are obtained from the integration over all the microstates of the system, and for the blocked fraction, the contributions $\left\langle\mu_{j}^{\|}\right\rangle_{B k}$ and $\left\langle\mu_{j}^{\perp}\right\rangle_{B k}$ (with $k=0,1$ ) have to be taken into account for each minimum (see Fig. 1). Summarizing, we have

$$
\begin{aligned}
& \left\langle\mu_{j}^{\|}\right\rangle=L_{j}\left\langle\mu_{j}^{\|}\right\rangle_{\mathrm{SP}}+\left(1-L_{j}\right) \sum_{k=0,1} P_{k}^{j}\left\langle\mu_{j}^{\|}\right\rangle_{B k}, \\
& \left\langle\mu_{j}^{\perp}\right\rangle=L_{j}\left\langle\mu_{j}^{\perp}\right\rangle_{\mathrm{SP}}+\left(1-L_{j}\right) \sum_{k=0,1} P_{k}^{j}\left\langle\mu_{j}^{\perp}\right\rangle_{B k},
\end{aligned}
$$

where $P_{k}^{j}(t)$ is the fraction of the ensemble represented by particle $j$ on the $k$ th minimum.

\section{Some details of the numerical calculations}

As the effects of the dipolar interactions are dependent on the magnetic configuration of the system, we start our calculation by considering a certain configuration $C(t)=$ $C\left[\boldsymbol{\mu}_{0}(t), \ldots, \boldsymbol{\mu}_{j}(t), \ldots, \boldsymbol{\mu}_{N-1}(t)\right]$ of the magnetic state of a chain of $N$ particles at time $t$. This means that we take into account the orientations of the magnetic moment for each NP. For all our treatment, we ignore the subscript that indicates the chain, since chains do not interact with each other and therefore this identification is unnecessary. To calculate the interaction field acting over particle $j$ (which does not depend on the particle itself) at time $t+\delta t$, we calculate $\mathbf{H}_{\mathrm{dip} j}$ according to the expressions in Eqs. (5) and (6) considering the previous configuration $C(t)$. Then, we let the magnetic state of the $j$ th particle evolve, obtaining a new configuration $C^{\prime}(t)=C\left[\boldsymbol{\mu}_{0}(t), \ldots, \boldsymbol{\mu}_{j}(t+\right.$ $\left.\delta t), \ldots, \boldsymbol{\mu}_{N-1}(t)\right]$. Because of hypothesis (f), we know that 
the evolution of the system between $t$ and $t+\delta t$ is sufficiently "smooth" as to maintain the energy profile for each NP, even when the influence of the dipolar field generated by the rest of the NPs is considered. This guarantees that the aforementioned process can be applied to our calculation. This procedure is repeated for the other particles; nevertheless, the interaction field is always calculated from the original configuration $C(t)$. The new total configuration $C(t+\delta t)$ is achieved when all particle moments have been updated, giving $C(t+\delta t)=C\left[\mu_{0}(t+\right.$ $\left.\delta t), \ldots, \boldsymbol{\mu}_{j}(t+\delta t), \ldots, \boldsymbol{\mu}_{N-1}(t+\delta t)\right]$. Consequently, our process ensures that the new configuration will not depend on the order in which the state of each particle is updated.

\section{RESULTS}

The magnetic properties of the particles used in the simulations are the anisotropy constant $K=5 \times 10^{3} \mathrm{~J} \mathrm{~m}^{-3}$ and the saturation magnetization $M_{S}=4 \times 10^{5} \mathrm{~A} \mathrm{~m}^{-1}$, which correspond to high-saturation maghemite magnetic nanoparticles [41]. We also assume a frequency $(f=$ $100 \mathrm{kHz})$ and a field amplitude $\left(H_{0}=32 \mathrm{kA} \mathrm{m}^{-1}\right)$ that are usual in MFH experiments, although we analyze how changing the frequency of the MFH experiment affects the relaxation in Sec. III B. The diameter of the NPs $\Phi$ is taken to be $25 \mathrm{~nm}$ and the distance between them $D$ is taken to be $40 \mathrm{~nm}$. Then, the dipolar field from neighboring particles goes approximately from 5 to $20 \mathrm{kA} \mathrm{m}^{-1}$.

The system is simulated at room temperature $(T=$ $293 \mathrm{~K})$. With these particle parameters and at room temperature, the particles are in the superparamagnetic regime in typical dc measurements and in the blocked regime in MFH conditions at zero field. The probability $L$ of being superparamagnetic will be modified by the applied field.

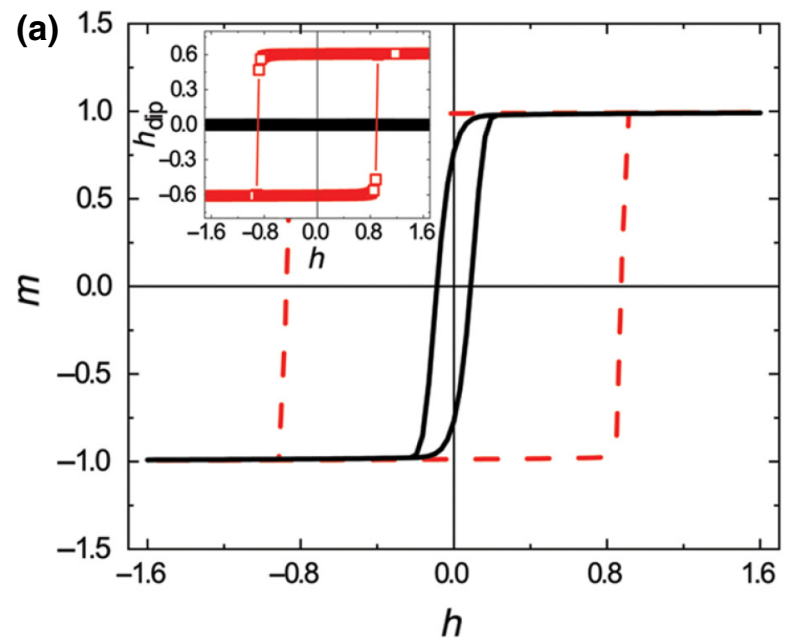

For our analysis, we normalize the magnetization $M$ and external applied field $H$ as $m=M / M_{S}$ and $h=H / H_{K}$.

To start exploring the effects of interparticle interactions in magnetic relaxation, we simulate chains oriented parallel to the external magnetic field $\left(\phi_{n}=0\right)$ and others oriented at $\phi_{n}=\pi / 4$ with a fixed length of $N=25 \mathrm{NPs}$ (the impact of varying the length of the chain is analyzed in Sec. III A). The results for the magnetization hysteresis loops of these systems are presented in Figs. 3(a) and 3(b), respectively, for the noninteracting and interacting cases. It is evident that dipolar interactions, in systems of this kind, increase the area $A$ enclosed by the loops for both orientations.

Another interesting feature arises from the analysis of both dipolar-field components. The insets in Fig. 3 show the parallel and perpendicular components of the dipolar field $\mathbf{H}_{\text {dip }}$ [with respect to the external magnetic field; see Fig. 2(c)] as a function of $h$ for $\phi_{n}=0$ and $\phi_{n}=\pi / 4$. These components are normalized by $M_{S}: h_{\text {dip }}^{\|}=H_{\text {dip }}^{\|} / M_{S}$, and $h_{\mathrm{dip}}^{\perp}=H_{\mathrm{dip}}^{\perp} / M_{S}$.

It is noticeable that both $h_{\text {dip }}$ and $m$ exhibit hysteresis in the same field region, as expected, with the exception of the null $h_{\mathrm{dip}}^{\perp}$ in the parallel orientation as a consequence of the symmetry of the problem. This implies that for the parallel orientation of the chain, the increase in the area of the magnetization hysteresis loop is caused exclusively by $H_{\text {dip }}^{\|}$. For this reason, it is easier to interpret the influence of dipolar interactions in the case of the parallel configuration, and we proceed to further analyze this particular situation in Sec. III C.

\section{A. Chains with different lengths}

The study of chains with different lengths has been discussed in several articles $[23,24,26]$. In any case, the

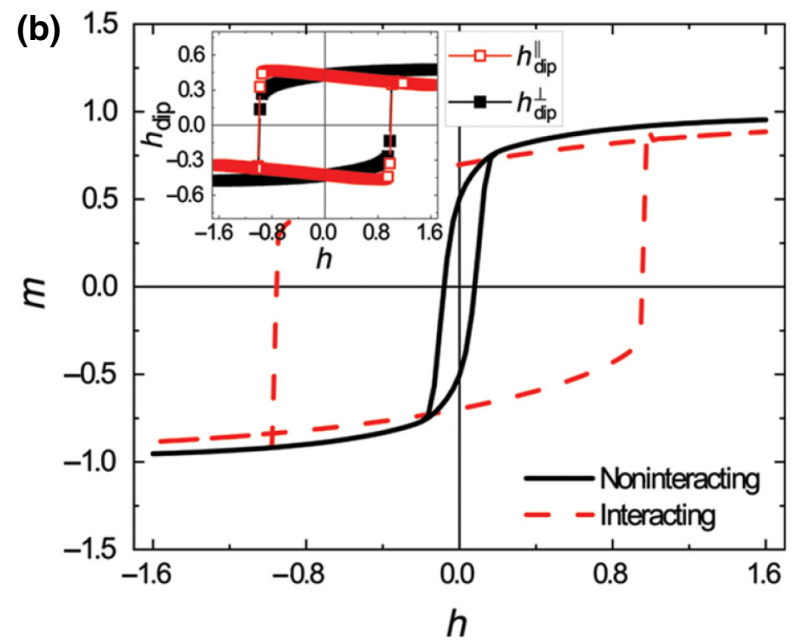

FIG. 3. Comparison of the magnetization hysteresis loops for the interacting and noninteracting cases corresponding to the (a) parallel $\left(\phi_{n}=0\right)$ and (b) $\phi_{n}=\pi / 4$ orientations. For the interacting case, the insets show the normalized components of the dipolar field as a function of the normalized external magnetic field. 


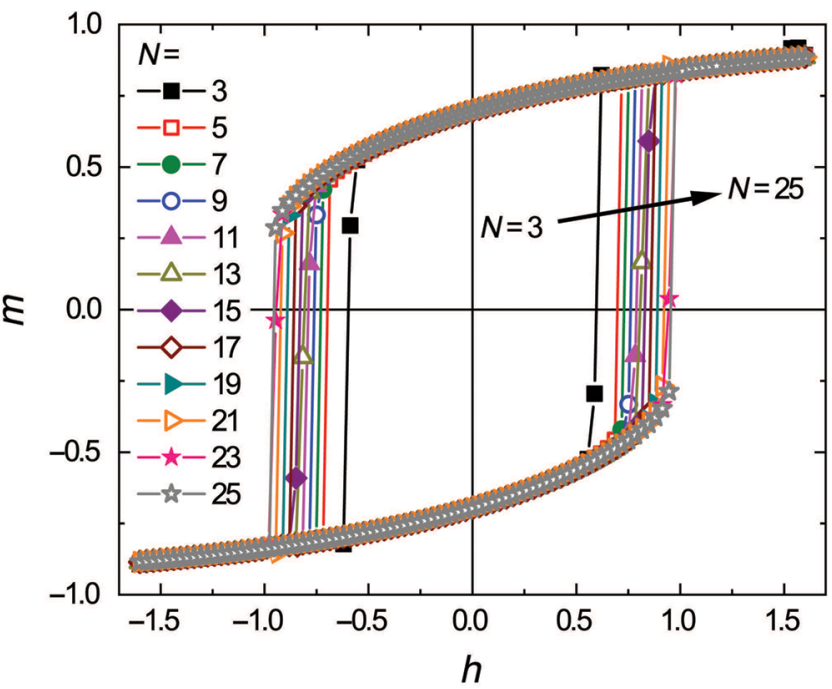

FIG. 4. Normalized magnetization as a function of the normalized external magnetic field for chains of variable length $N$ with orientation $\phi_{n}=\pi / 4$. The arrow indicates the direction in which $N$ increases.

simulations of chains are also performed with variable length $N$ in different orientations with respect to the applied field. In Fig. 4 we show the results for the average $m(h)$ loops in the particular case of $\phi_{n}=\pi / 4$, but the behavior is the same for other orientations.

It is clearly noticeable that for very short chains $(N=3$ and 5) the average loop exhibits a lower coercivity than for the longer chains $(N \geq 7)$ due to border effects being an important contribution in the averaging. This feature saturates for the longer chains, in accordance with several previous reports $[23,24,26]$. Because of this "asymptotic saturation" behavior, $N=25$ is used as the length of all the chains simulated for the results shown henceforth. In this situation, border effects do not affect the average loops significantly.

\section{B. Influence of the frequency of the experiment $f$ on the $m(h)$ loops}

We have been studying the relaxation of NPs and chains under a fixed frequency $f$ of $100 \mathrm{kHz}$. However, it is of utmost importance to know if there are significant changes when $f$ is varied when one is designing a MFH experiment. When one is designing an experiment, the experimental working conditions may be chosen taking into account the particularities of the NP system and the application. If we are talking of MFH as a therapy, there are several proposed therapeutical limits $H_{0} f[42,43]$ that restrict the choice of the experimental conditions and therefore need to be considered.

In Fig. 5(a), we compare the previous loops (at $f=$ $100 \mathrm{kHz}$ ) with ones obtained at a much higher frequency, $f=1 \mathrm{MHz}$; that is, the conventional upper limit in the

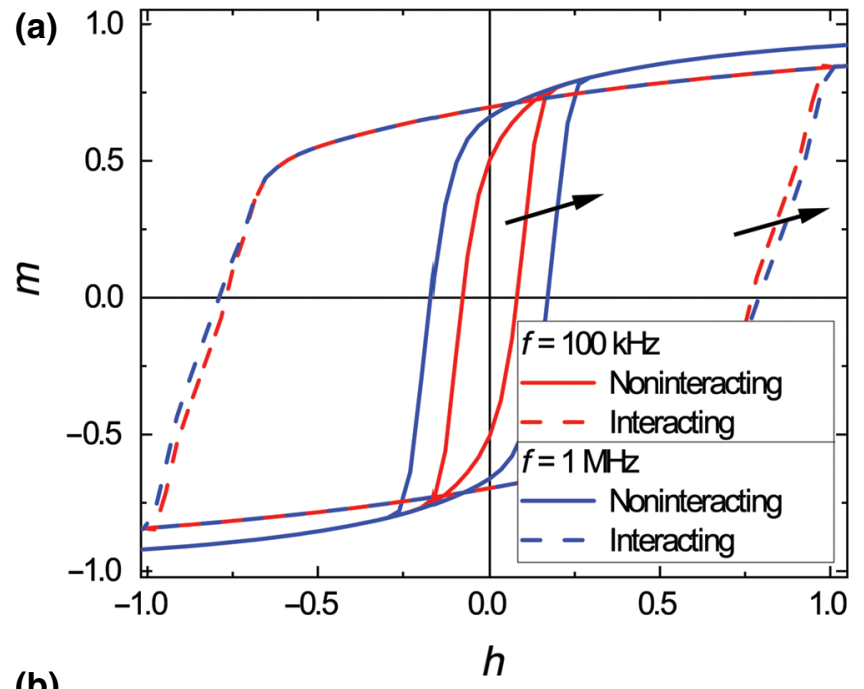

(b)

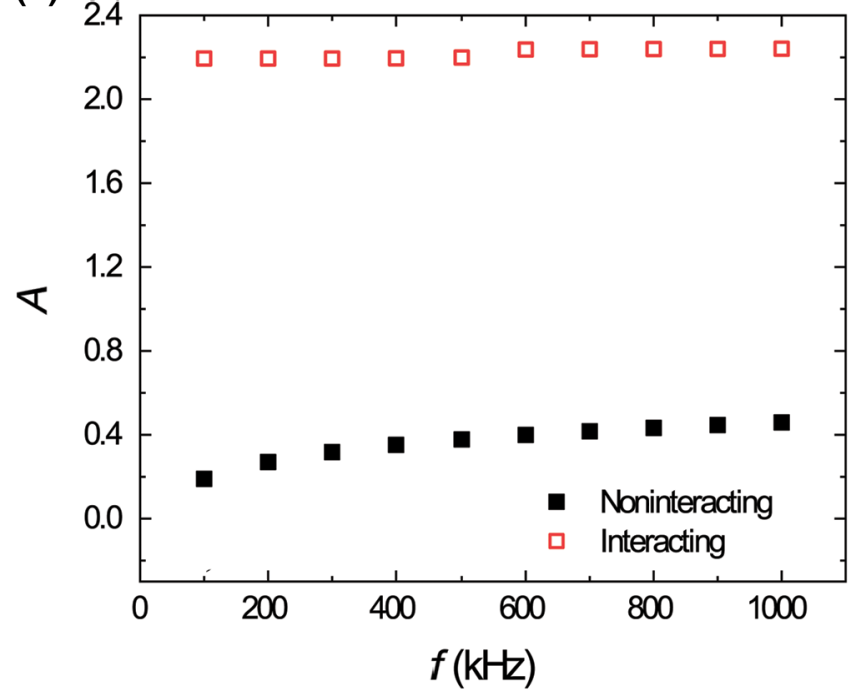

FIG. 5. (a) Normalized magnetization as a function of the normalized external magnetic field for chains with orientation $\phi_{n}=\pi / 4$ exposed to an ac field of different frequencies $f$. The arrows indicate the direction in which $f$ increases. (b) Dimensionless area $A$ of the $m(h)$ loops [as the ones shown in (a) for two frequencies] as a function of $f$.

field of MFH application and instrumentation. Noninteracting and interacting (chains) systems are both considered. The effect of increasing the frequency is to increase the enclosed area $A$ for the noninteracting systems. Meanwhile, for the case of interacting NP chains, a barely noticeable effect on the coercive field $H_{C}$ is seen.

We simulate noninteracting and interacting systems spanning the whole MFH frequency range $(100 \mathrm{kHz}$ to $1 \mathrm{MHz}$ ). The results are shown in Fig. 5(b), where the dimensionless area $A$ [the area calculated from the normalized $m(h)$ loops] is plotted as a function of $f$. Again, we notice that the area of the interacting loops stays practically the same, while the noninteracting loops increase their area at higher frequencies but reach saturation in this frequency range. 


\section{Effect of the dipolar field in the hysteresis loops}

Returning to the task of determining the influence of the dipolar-field components on the magnetization hysteresis loops, it is essential to assess the problem as selfconsistent, as the magnetization hysteresis loop generates hysteresis in the dipolar field and the dipolar field corrects the magnetization. For each value of $H$, self-consistency is attained in the numerical simulations and, equivalently, dynamic equilibrium is reached in the experiment. The problem is solved by our making the field sweep step small enough as to not affect the magnetization (and therefore the resulting dipolar field) significantly, except in the case of magnetic moment reversal. In practice, this is achieved by our verifying that the discretization of the field sweep does not modify the appearance of the hysteresis loop and, as a consequence, its enclosed area.

In Fig. 6, the results of a hypothetical experiment in the parallel configuration are shown to illustrate the mechanism through which interactions produce an increase of the loop-enclosed area. For this configuration, only $H_{\text {dip }}^{\|}$needs to be taken into account, simplifying the analysis of the problem. As mentioned in the model description, interactions do not change the intrinsic coercive field of the NPs but they produce a shift in the local field $\mathbf{H}_{\text {loc }}=\mathbf{H}+\mathbf{H}_{\text {dip }}^{\|}$ on each particle. The hysteresis loop of a given interacting particle as a function of $H_{\text {loc }}$ [see Fig. 6(c)] will look exactly the same as the one associated with a noninteracting particle as a function of $H$ [see Fig. 6(a)]. However, when the magnetization for the interacting case is plotted as a function of the external field (as done in a real experiment), an increase in coercivity will be evident, as in Fig. 6(d).

The dipolar field [see Fig. 6(b)], generates a shift $\Delta H$ in the local field with respect to the external field. This can be clearly visualized in Fig. 6 for the ascending branch of the hysteresis loops, where solid circles represent a given value of $H$ and open circles represent the corresponding $H_{\text {loc }}$ for the interacting system. By symmetry, an analogous situation is observed for the descending branch of the hysteresis loops, yielding an increased coercivity.

The aforementioned ideas allow us to explain the effect of $H_{\text {dip }}^{\|}$on the hysteresis given that $H_{\text {dip }}^{\perp}$ is zero for the parallel configuration. The role of the perpendicular component for other orientations of the chain remains to be seen. It is relevant to study what the individual contributions of both components are for different orientations as well as in randomly oriented chains. In this way we can determine if $H_{\text {dip }}^{\perp}$ produces an effect to be considered in future magnetic relaxation models.

\section{Evolution of the hysteresis loops with the orientation of the chain}

For simplicity, we have analyzed the parallel configuration so far, but to determine the contribution of $H_{\text {dip }}^{\perp}$, we



FIG. 6. Interaction effects on the magnetic response of a system with only a parallel dipolar component (i.e., $\phi_{n}=0$ ). (a) Magnetization of a noninteracting particle and (b) parallel component of the dipolar field as a function of the local magnetic field $\mathbf{H}_{\mathrm{loc}}=\mathbf{H}+\mathbf{H}_{\text {dip }}^{\|}$. (c) Magnetization of an interacting particle as a function the local field. (d) Loop given in (c) but plotted as a function of the external magnetic field $H$. A solid circle represents a given $H$ value and an open circle represents its corresponding $H_{\text {loc }}$ value.

need to study chains in other orientations. In Fig. 7, we present magnetization hysteresis loops for chains in different orientations, as well as the dipolar-field components as a function of $h$. Looking at the parallel and perpendicular components of the dipolar field as a function of the applied field in Figs. 7(b) and 7(c) respectively, we can see that hysteresis is again exhibited. For the perpendicular component, for $\phi_{n} \neq 0$, we have $h_{\text {dip }}^{\perp} \neq 0$, and when $\phi_{n}$ approaches $\pi / 2$, the enclosed area of the $m(h)$ loop gets smaller.

There are more effects apart from the reduction of the area: the hysteresis loops of the dipolar components change not only their shape but also their distribution in 


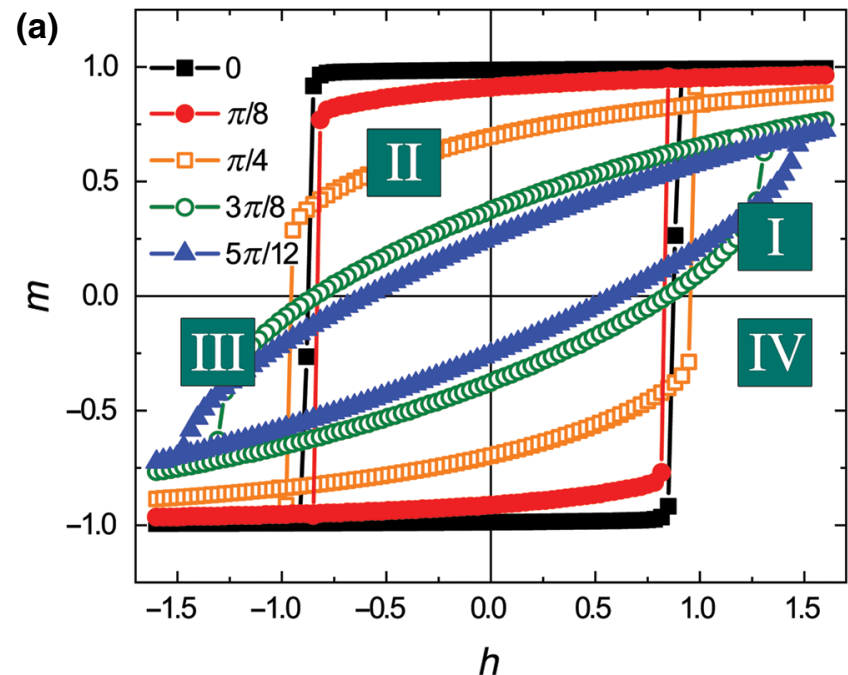

(b)


FIG. 7. Normalized (a) magnetization and components of the dipolar field (b) $h_{\text {dip }}^{\|}$and (c) $h_{\text {dip }}^{\perp}$ as a function of the normalized external field for orientations $0 \leq \phi_{n}<\pi / 2$. The quadrant numbers are given in boxes. each quadrant. Independently of the corresponding orientation, most of the $m(h)$ loop-enclosed area is always in quadrants I and III [see Fig. 7(a) for reference]. For $h_{\text {dip }}^{\perp}(h)$ loops, a similar behavior is observed. Nevertheless, for $h_{\text {dip }}^{\|}(h)$, the loops shift from quadrants I and III to quadrants II and IV as the orientation nears $\pi / 2$. A detailed study of how the changes in the shape and distribution of the $h_{\mathrm{dip}}^{\|}(h)$ and $h_{\mathrm{dip}}^{\perp}(h)$ loops influence changes in the $m(h)$ loop is presented in the Appendix.

\section{E. Role of the parallel and perpendicular components of the dipolar field as a function of the orientation of the chain}

The problem of evaluating the separate influence of each component, $h_{\mathrm{dip}}^{\|}$and $h_{\mathrm{dip}}^{\perp}$, is complicated because they both act simultaneously in the simulation, as well as in experiments performed in the same conditions. In other words, during the evolution of the hysteresis-loop measurement or calculation, the changes in the magnetization affect the dipolar components dynamically. In turn, the components also affect the evolution of the magnetization. This is why (in the framework of this effective "interaction" between $H_{\text {dip }}^{\|}$and $H_{\text {dip }}^{\perp}$ through $M$ ) it is complex to clearly dissociate the role of each component for a given orientation of the chain.

For our analysis, we take advantage of the control over each component that we have in simulations: although in the experiment there is no possibility of switching an individual component off, in the simulation we can do it and it can be a powerful tool for the thorough understanding of the problem. However, because of the self-consistency we mentioned earlier, it is not sufficient to ignore one of the $\mathbf{H}_{\text {dip }}$ components and to evaluate the effect of the other on the increase of the area of the loops. Thus, we implement a protocol to correctly assess this problem. Assuming that we are interested in studying the influence of the $H_{\text {dip }}^{\|}$ and $H_{\text {dip }}^{\perp}$ components as a function of the orientation of the chain $\phi_{n}$, for each value of $\phi_{n}$, two hysteresis loops are simulated: one of them with both of the components (with enclosed area $A_{T}$ ) and the other in which we exclude the component $\left(H_{\text {dip }}^{\|}\right.$or $\left.H_{\text {dip }}^{\perp}\right)$ that we are interested in (with enclosed area $A_{\perp}$ or $A_{\|}$, respectively). Then, for each $\phi_{n}$, we can calculate

$$
\begin{aligned}
& a_{\|}\left(\phi_{n}\right)=\frac{A_{T}\left(\phi_{n}\right)-A_{\perp}\left(\phi_{n}\right)}{A_{T}\left(\phi_{n}\right)}, \\
& a_{\perp}\left(\phi_{n}\right)=\frac{A_{T}\left(\phi_{n}\right)-A_{\|}\left(\phi_{n}\right)}{A_{T}\left(\phi_{n}\right)} .
\end{aligned}
$$

In this way, $a_{\|}$and $a_{\perp}$ give us relative information about the increase in the area of the magnetization loop related to the respective component of the dipolar field. In addition, following the same idea as for the expressions in 
Eq. (7), we consider the parameter $a_{T}\left(\phi_{n}\right)=\left[A_{T}\left(\phi_{n}\right)-\right.$ $\left.A_{0}\left(\phi_{n}\right)\right] / A_{T}\left(\phi_{n}\right)$, where $A_{0}$ the enclosed area for the noninteracting case, to analyze the influence of both components at the same time.

In Fig. 8(a), we present the results for $a_{T}, a_{\|}$, and $a_{\perp}$ as a function of $\phi_{n}$. It is important to note that $\phi_{n}$ is varied up to $4 \pi / 9$ because above this value crossover [44] of the hysteresis branches, which is inherent to the Stoner-Wohlfarth model [35], occurs.

To get a better understanding of this issue, we start by analyzing $\phi_{n}=0$. In this case, $H_{\mathrm{dip}}^{\perp}=0$, which causes $A_{T}(0)=A_{\|}(0)$, finally giving $a_{\perp}(0)=0$ and $a_{T}(0)=$ $a_{\|}(0)$ [see Fig. 8(a)]. This reflects the fact that $H_{\text {dip }}^{\perp}$ has no effect in the parallel configuration (as observed in the insets in Fig. 3) and that all contributions come from $H_{\text {dip }}^{\|}$, as previously noted.

Our results show opposite behaviors between $a_{\|}\left(\phi_{n}\right)$ and $a_{\perp}\left(\phi_{n}\right)$ when $\phi_{n}$ approaches $\pi / 2$. The contribution
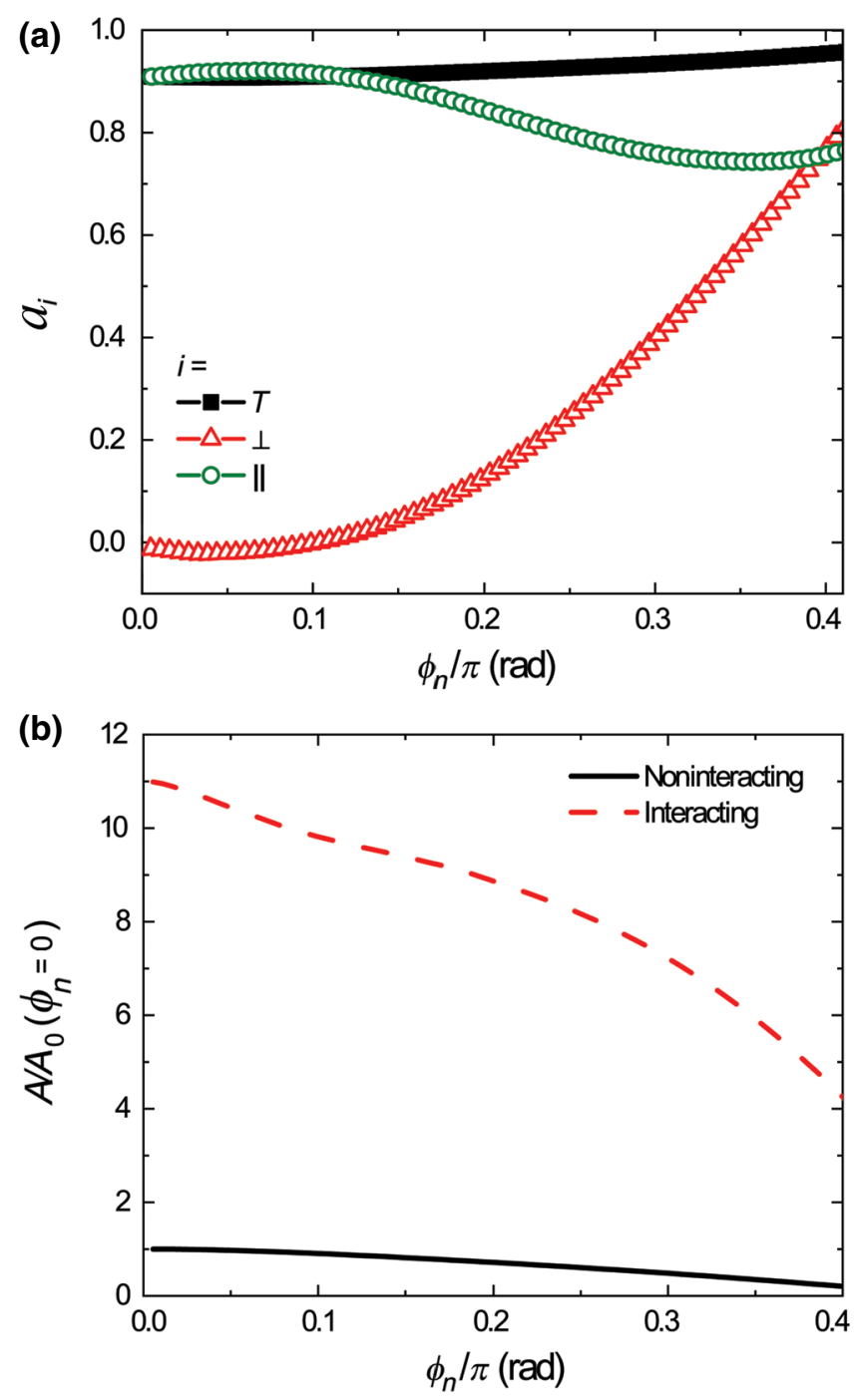

FIG. 8. (a) Relative increases of the area $a_{T}, a_{\|}$, and $a_{\perp}$ and (b) normalized absolute area $A$ as a function of $\phi_{n}$. of $H_{\mathrm{dip}}^{\perp}$ increases, while that of $H_{\mathrm{dip}}^{\|}$decreases. However, for all orientations, the total effect of the components is equal to or greater than that of each one separately $\left[a_{T}\left(\phi_{n}\right) \geq a_{\|}\left(\phi_{n}\right), a_{\perp}\left(\phi_{n}\right)\right]$ and the effect of both components is not a simple addition of each one $\left[a_{T}\left(\phi_{n}\right) \leq\right.$ $\left.a_{\|}\left(\phi_{n}\right)+a_{\perp}\left(\phi_{n}\right)\right]$. In conclusion, the influence of the parallel (perpendicular) component is more important near the parallel (perpendicular) orientation of the chain. This stresses the importance of considering that $\mathbf{H}_{\text {dip }}$ can have a component perpendicular to $\mathbf{H}$ when one is modeling chains near the perpendicular orientation, which is not always done.

As $a_{\perp} \geq 0$ and $a_{\|} \geq 0$ for all $\phi_{n}$, there is no way that the net effect of having both components can be detrimental to the increase of the hysteresis area (i.e., the SPA) in the systems considered. As $a_{T} \geq 0$, and we can affirm that in low-anisotropy chains of NPs of this type, interactions always help to increase the hysteresis area.

In Fig. 8(a) it is observed that interactions (in the case that both components of the dipolar field are considered) have a greater relative influence for orientations near $\phi_{n}=$ $\pi / 2$. However, the important parameter that determines the SPA in a MFH experiment is the absolute area $A$, which we plot in Fig. 8(b) as a function of $\phi_{n}$. We normalize the absolute enclosed area $A$ to the area of the noninteracting system in the parallel configuration $A_{0}\left(\phi_{n}=0\right)$. With this in mind, we conclude that the strong increase of $a_{\perp}$ approaching $\phi_{n}=\pi / 2$ is a direct consequence of the reduction of the absolute hysteresis area. Also, it is now evident that the most-favorable case in terms of SPA occurs when the chain is parallel to the external field.

\section{F. Randomly oriented chains}

Because of the possibility of getting randomly oriented chains inside a cell in a MFH experiment, chains in this condition are simulated. To accelerate the calculations, we use the azimuthal symmetry of the problem, discretizing the values of the polar angle $\phi_{n}$ of the orientation of each chain and weighting the value of the corresponding area by the solid angle $\left(\sin \phi_{n}\right.$ ), avoiding unnecessary sweeps of the azimuthal angle. In this particular case, we take 91 chains with consecutive, equally spaced orientations $0<$ $\phi_{n}<\pi / 2$. Hysteresis loops with and without interactions are obtained. Loops where one of the dipolar-field components is forced to zero are also simulated for comparison [see Fig. 9(a)].

For all cases considered, the interactions help to increase the area of the loops compared with the noninteracting case. It is noticeable that a system with only $H_{\text {dip }}^{\|}$produces a bigger loop than a system with only $H_{\text {dip }}^{\perp}$ considered. By the definition of the solid angle, we have more chains oriented near $\phi_{n}=\pi / 2$ than near the parallel configuration. However, the phenomenon can be explained because, as we pointed out before, the absolute area of the loops 


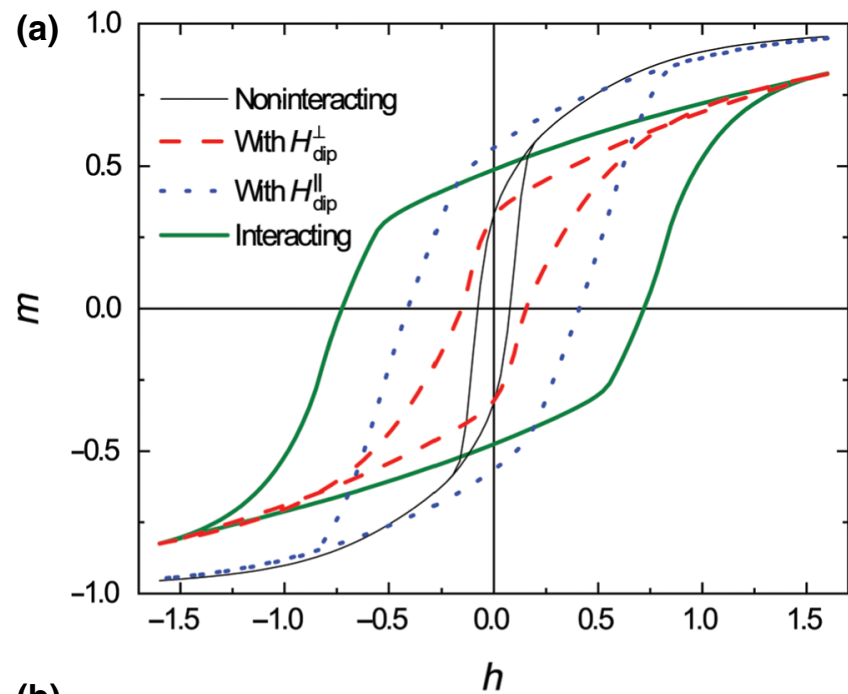

(b)



FIG. 9. (a) Normalized magnetization as a function of the normalized external magnetic field for randomly oriented chains. The loops without interactions considered (0), with both dipolar-field components considered $(\perp+\|)$, and with only one dipolar-field component considered ( $\perp$ or $\|$ ) are presented. (b) Normalized absolute hysteresis area for the aforementioned randomly oriented chains and chains in the parallel configuration, both noninteracting ( 0$)$ and interacting $(\perp$ or $\|) . A_{0}\left(\phi_{n}=0\right)$ is the area for the noninteracting case in the parallel configuration.

corresponding to chains near the parallel configuration is considerably bigger than that of those near $\phi_{n}=\pi / 2$ [see Fig. 8(b)].

Moreover, it is important to note that a model for interacting systems considering only the interaction component parallel to the external field underestimates the area enclosed by the loops and results in a wrong description of the evolution of hysteresis loops as a function of $H$.

In Fig. 9(b), the area of the loops for the randomly oriented chains in Fig. 9(a) is compared with the area for chains parallel to the external ac field for the noninteracting case and the interacting case. It is remarkable that interactions can make up for the nonalignment of the chains, as the hysteresis area for the randomly oriented interacting chains $(L+\|)$ is considerably larger than the area for the noninteracting chains and is comparable to that for the most-favorable case. This implies that the formation of chains in a MFH experiment can considerably improve the performance in terms of the SPA, even if they are not aligned.

\section{CONCLUSIONS}

In the case of ideal systems of low-anisotropy $\left(H_{K}<\right.$ $H_{0}$ ) chains constituted by uniaxial NPs in standard MFH experimental situations $\left(f=100 \mathrm{kHz}, H_{0}=32 \mathrm{kA} \mathrm{m}^{-1}\right.$, and $T=293 \mathrm{~K}$ ), we determine that interactions always help to increase the area of $m(h)$ compared with the system with noninteracting particles for specific orientations and also for randomly oriented chains. This increase of the area implies a higher SPA, which gives better performance in hyperthermia experiments. We interpret this increase in the area enclosed by the loops as the result of a shift between the local field at the particles' site and the external applied field.

We show that varying the frequency of the experiment in the range of MFH applications ( $100 \mathrm{kHz}$ to $1 \mathrm{MHz}$ ) does not affect the enclosed hysteresis area significantly. Moreover, the loops corresponding to noninteracting systems present a small enclosed-area increase with increasing $f$ that saturates in the range studied, while the interacting loops are barely modified near $H_{C}$.

We study the dipolar field by decomposing it into two components: one parallel and the other perpendicular to the external field. Simulations of chains in different orientations show hysteresis of both components of the dipolar field with respect to the external applied field. In terms of area (i.e., SPA), the most-favorable case is the chain parallel to the external field.

We find a strong correlation between the distribution of the hysteresis loops in the quadrants (that of the components of the dipolar fields with respect to the magnetization loop) with the influence of these components on the area enclosed by the magnetization hysteresis loop (as presented in the Appendix). This result reinforces our idea that the dipolar field (both components) produces a shift in the magnetization response that results in an increase in the area enclosed by the magnetization hysteresis loop.

We conclude that it is essential to include the perpendicular dipolar component in a model when chains oriented near the perpendicular configuration are taken into account, because this component determines the magnetic behavior of the system. Near the perpendicular direction, the relative influence of interactions in the area of the magnetization hysteresis loops is larger than in the parallel direction. However, taking into account the absolute 
influence, we find the best scenario is when the chains are oriented parallel to the external field, due to the larger area of the hysteresis loop in this condition. In the parallel and perpendicular configurations, the corresponding component is the one that contributes the most to the increase in the area of the magnetization hysteresis loop of the system.

Finally, in our study of randomly oriented chains we find that if the formation of chains can be somehow promoted, the performance of these chains in MFH experiments will be significantly better than that of a noninteracting dispersed-particle system, even if the chains are not aligned with the external ac field (i.e., if they are randomly oriented).

\section{ACKNOWLEDGMENTS}

The authors thank Dr. Alejandro Butera for his thorough reading of the manuscript and his valuable advice that helped to improve it. This work is part of a research project supported by Agencia Nacional de Promoción Científica y Tecnológica (Argentina) under Grants No. PICT-20122995 and No. PICT-2015-0883, CONICET. We thank Universidad Nacional de Cuyo for supporting us with project SIIP No. 06/C564.

\section{APPENDIX}

\section{Study of the evolution of $m(h), h_{\text {dip }}^{\|}(h)$, and $h_{\text {dip }}^{\perp}(h)$ loops with the orientation of the chain and its correlation with the hysteresis area}

In the particular case of the parallel configuration, we see that the dipolar field generates a shift in the local field that produces an increase of the hysteresis. We also study the effect of each component of the dipolar field on the hysteresis of the magnetization for different $\phi_{n}$. On the other hand, the hysteresis loops show us that there are important changes in their shape and distribution as we change the orientation of the chain with respect to the external field. Here we quantify the similarity between the loops of each component of the dipolar field $h_{\text {dip }}$ and the magnetization loop and correlate it with the effect on the enclosed area.

As we see from Fig. 7, what we call the "distribution" of the $h_{\text {dip }}^{\|}(h)$ or $h_{\text {dip }}^{\perp}(h)$ loop (i.e., in which quadrants the majority of the enclosed area is found) is not always the same and it depends on the orientation of the chain, $\phi_{n}$. The $h_{\text {dip }}^{\perp}(h)$ loop is distributed in the usual fashion [as an $m(h)$ loop, in quadrants I and III]. However, the $h_{\text {dip }}^{\|}(h)$ loop experiences a "transition": it starts distributed as usual and for $\phi_{n} \approx 3 \pi / 8$, it shifts to quadrants II and IV.

To determine what influence the changes in the distribution of the dipolar-component loops have on the magnetization hysteresis loop of the chain, we need a way of quantifying them. For that, we conveniently define two parameters, $C^{\|}$and $C^{\perp}$, given by



FIG. 10. (a) $C^{i}$ and (b) its derivative $d C^{i} / d \phi_{n}$ as a function of $\phi_{n}$. (c) Magnetization and dipolar-component- $i$ loops when $C^{i} \sim$ 1 or $C^{i} \sim-1, i=\|, \perp$. The quadrants that enclose most of the hysteresis area for each loop are grayed.

$$
\begin{array}{r}
C^{\|}=\frac{\sum_{i} m^{+}\left(h_{i}\right) h_{\mathrm{dip}}^{\|+}\left(h_{i}\right)\left|m^{-}\left(h_{i}\right) h_{\mathrm{dip}}^{\|-}\left(h_{i}\right)\right|}{\sum_{i}\left|m^{+}\left(h_{i}\right) h_{\mathrm{dip}}^{\|+}\left(h_{i}\right) m^{-}\left(h_{i}\right) h_{\mathrm{dip}}^{\|-}\left(h_{i}\right)\right|}, \\
C^{\perp}=\frac{\sum_{i} m^{+}\left(h_{i}\right) h_{\mathrm{dip}}^{\perp+}\left(h_{i}\right)\left|m^{-}\left(h_{i}\right) h_{\mathrm{dip}}^{\perp-}\left(h_{i}\right)\right|}{\sum_{i}\left|m^{+}\left(h_{i}\right) h_{\mathrm{dip}}^{\perp+}\left(h_{i}\right) m^{-}\left(h_{i}\right) h_{\mathrm{dip}}^{\perp-}\left(h_{i}\right)\right|},
\end{array}
$$

where the general magnitude $x^{+}\left(h_{i}\right)\left[x^{-}\left(h_{i}\right)\right]$, with $x=$ $m, h_{\text {dip }}^{\|}$, or $h_{\text {dip }}^{\perp}$, is defined as $x^{+}\left(h_{i}\right)=x^{\text {sup }}\left(h_{i}\right)+x^{\inf }\left(h_{i}\right)$ $\left[x^{-}\left(h_{i}\right)=x^{\text {sup }}\left(h_{i}\right)-x^{\text {inf }}\left(h_{i}\right)\right]$, where $x^{\text {sup }}\left(h_{i}\right)\left[x^{\inf }\left(h_{i}\right)\right]$ is the value of $x\left(h_{i}\right)$ for the descending (ascending) branch of the corresponding loop.

The idea in Eq. (A1) can be understood if we decompose a hysteresis loop into the descending branch $m^{\text {sup }}(h)$ (going from $H_{0}$ to $-H_{0}$ ) and the ascending branch $m^{\inf }(h)$ (going from $-H_{0}$ to $H_{0}$ ). We take an arbitrary field $h_{i}$ and calculate the difference between the descending branch and the ascending branch [given by $m^{-}\left(h_{i}\right)=m^{\text {sup }}\left(h_{i}\right)-$ $\left.m^{\inf }\left(h_{i}\right)\right]$ and their sum $\left[m^{+}(h)=m^{\text {sup }}\left(h_{i}\right)+m^{\inf }\left(h_{i}\right)\right]$ and then compute their product $\left[\mathrm{m}^{+}\left(h_{i}\right) \mathrm{m}^{-}\left(h_{i}\right)\right]$. The same procedure is conducted with the dipolar component of interest, $h_{\text {dip }}^{\|}$or $h_{\text {dip }}^{\perp}$, giving $h_{\text {dip }}^{\|-}\left(h_{i}\right)\left[h_{\text {dip }}^{\perp-}\left(h_{i}\right)\right], h_{\text {dip }}^{\|+}\left(h_{i}\right)$ $\left[h_{\text {dip }}^{\perp+}\left(h_{i}\right)\right]$, and their product. If $m^{\text {sup }}\left(h_{i}\right)>\left|m^{\inf }\left(h_{i}\right)\right|$, this means that in a neighborhood of $h_{i}$, the loop occupies a greater fraction in the upper quadrants and consequently $m^{+}\left(h_{i}\right) m^{-}\left(h_{i}\right)>0$. Multiplying this factor by $h_{\text {dip }}^{\|+}\left(h_{i}\right) h_{\text {dip }}^{\|-}\left(h_{i}\right)\left[h_{\text {dip }}^{\perp}\left(h_{i}\right) h_{\text {dip }}^{\perp-}\left(h_{i}\right)\right]$, we can compare the behavior of the loop of the dipolar component in question with that of the magnetization at a point $h_{i}$. This is 
(a)

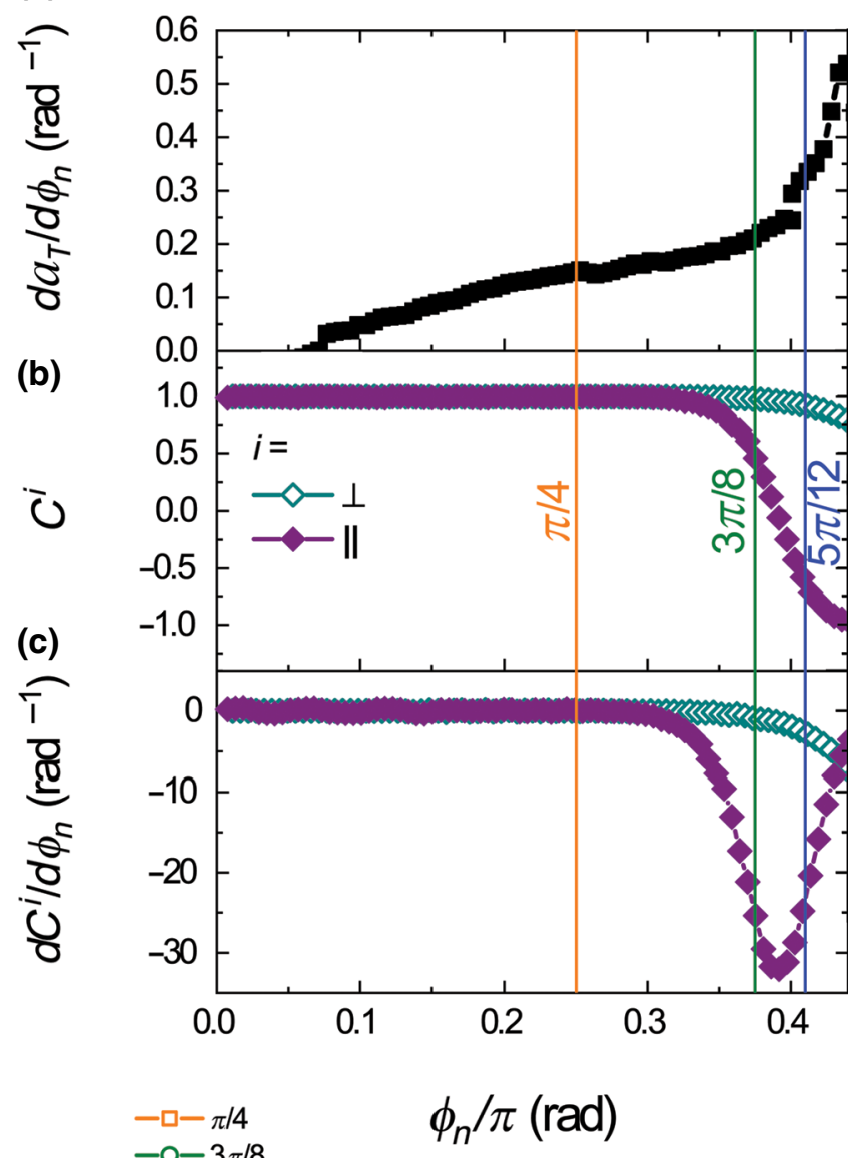

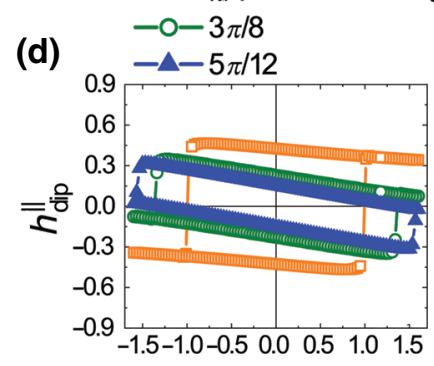

$h$ (e)

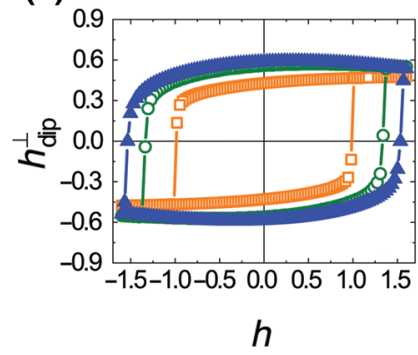

FIG. 11. (a) Derivative of $a_{T}=\left(A_{T}-A_{0}\right) / A_{T}$ ( $A_{0}$ being the enclosed area in the noninteracting case) with respect to $\phi_{n}$, (b) $C^{\|}$and $C^{\perp}$ and (c) their derivatives with respect to $\phi_{n}$ as a function of $\phi_{n}$. The vertical lines in (b),(c) denote the orientations for which loops are presented in (d),(e). Dipolar-component (d) $h_{\text {dip }}^{\|}$and (e) $h_{\text {dip }}^{\perp}$ loops for different orientations.

the essence behind the expressions in Eq. (A1), calculated for every $h_{i}$ and summed up. The denominator in the expressions is only a normalization to produce $C^{\|} \in$ $[-1 ; 1]$ and $C^{\perp} \in[-1 ; 1]$, since $m(h)$ and $h_{\text {dip }}(h)$ can have very different absolute values.

Finally, the values assumed by $C^{\|}$and $C^{\perp}$ are simple to interpret: $C^{\|}\left(C^{\perp}\right)$ conveniently indicates when the loop $h_{\text {dip }}^{\|}\left(h_{\text {dip }}^{\perp}\right)$ is distributed as the $m(h)$ loop $\left(C^{\|}, C^{\perp}=1\right)$ or opposite to it $\left(C^{\|}, C^{\perp}=-1\right)$, as schematized in Fig. 10. To visualize changes in the distribution with $\phi_{n}$ more easily, it is necessary to derive $C^{\|}$and $C^{\perp}$ with respect to $\phi_{n}$ [see Fig. 10(b)].

Moving on to the results for chains with different orientations, in Fig. 11(a), we present the derivative of $a_{T}=$ $\left(A-A_{0}\right) / A$ with respect to $\phi_{n}$ as a function of $\phi_{n}$. This graph represents the derivative of the $a_{T}$ curve in Fig. 8(a). In Figs. 11(b) and 11(c) we show $C^{\|}$and $C^{\perp}$ and the derivatives $d C^{\|} / d \phi_{n}$ and $d C^{\perp} / d \phi_{n}$ as a function of $\phi_{n}$.

The correlation between the $d a_{T} / d \phi_{n}, d C^{\|} / d \phi_{n}$ and $d C^{\perp} / d \phi_{n}$ curves is clear. Both $d a_{T} / d \phi_{n}$ and $d C^{\|} / d \phi_{n}$ show strong changes for $\phi_{n} \gtrsim 0.35 \pi$ that are correlated with the change in the distribution of the $h_{\text {dip }}^{\|}(h)$ loop, shown in Fig. 11(d): $d a_{T} / d \phi_{n}$ starts to increase significantly when the $h_{\mathrm{dip}}^{\|}(h)$ loop has a "transition" from quadrants I and III to quadrants II and IV.

For higher $\phi_{n}$ values [when $d C^{\|} / d \phi_{n}=0$, so $h_{\text {dip }}^{\|}(h)$ no longer changes its area distribution], the perpendicular component starts to play a role. $d C^{\perp} / d \phi_{n}$ slightly decreases, meaning it could be responsible for the increase of $d a_{T} / d \phi_{n}$, and as consequence the increase of $a_{T}$, at orientations near the perpendicular one. However, the change in the distribution of $h_{\text {dip }}^{\perp}(h)$ is relatively small [as it cannot be clearly seen in the loops in Fig. 11(e)]. From Fig. 8(a) we infer that the relative influence of the perpendicular component $h_{\mathrm{dip}}^{\perp}$ on the magnetization loop is stronger than that of $h_{\mathrm{dip}}^{\|}$as the orientation becomes closer to $\phi_{n}=\pi / 2$. However, this influence could be an effect due to not only the slight change of the distribution of the loop but also an increase in the area of the $h_{\mathrm{dip}}^{\perp}(h)$ loop, as can be inferred from Fig. 11(e).

With these results, we conclude that the behavior of $a_{T}$ as a function of $\phi_{n}$ is closely linked to how the shape and distribution of the $h_{\mathrm{dip}}^{\|}(h)$ and $h_{\mathrm{dip}}^{\perp}(h)$ loops change with reference to $m(h)$. Those changes are more pronounced when $\phi_{n}$ approaches $\pi / 2$. First, for $\phi_{n}$ in the range from $0.35 \pi$ to $0.45 \pi$, the effect of $h_{\text {dip }}^{\|}$dominates the evolution of the loops [a result that complements what is observed in Fig. 8(a)] and then the contribution of $h_{\mathrm{dip}}^{\perp}(h)$ could cause $a_{T}$ to keep increasing (regardless of the parallel-component loop maintaining its distribution intact).

[1] S. Laurent, S. Dutz, U. O. Häfeli, and M. Mahmoudi, Magnetic fluid hyperthermia: Focus on superparamagnetic iron oxide nanoparticles, Adv. Colloid Interface Sci. 166, 8 (2011).

[2] P. Das, M. Colombo, and D. Prosperi, Recent advances in magnetic fluid hyperthermia for cancer therapy, Colloids Surf. B 174, 42 (2019).

[3] C. S. Kumar and F. Mohammad, Magnetic nanomaterials for hyperthermia-based therapy and controlled drug delivery, Adv. Drug Deliv. Rev. 63, 789 (2011). 
[4] A. Silva, T. Oliveira, J. Mamani, S. Malheiros, L. Malavolta, L. Pavon, T. Sibov, E. Amaro, A. Tannús, E. Vidoto, M. Martins, R. Santos, and L. Gamarra, Application of hyperthermia induced by superparamagnetic iron oxide nanoparticles in glioma treatment, Int. J. Nanomed. 6, 591 (2011).

[5] N. A. Usov and B. Y. Liubimov, Dynamics of magnetic nanoparticle in a viscous liquid: Application to magnetic nanoparticle hyperthermia, J. Appl. Phys. 112, 023901 (2012).

[6] I. Khan, K. Saeed, and I. Khan, Nanoparticles: Properties, applications and toxicities, Arab. J. Chem. 12, 908 (2019).

[7] B. R. Jarrett, M. Frendo, J. Vogan, and A. Y. Louie, Size-controlled synthesis of dextran sulfate coated iron oxide nanoparticles for magnetic resonance imaging, Nanotechnology 18, 035603 (2007).

[8] K. Wang, W. Tan, and X. He, in 2005 IEEE Engineering in Medicine and Biology 27th Annual Conference (2005), pp. 717-719.

[9] E. De Biasi, C. A. Ramos, R. D. Zysler, and H. Romero, Large surface magnetic contribution in amorphous ferromagnetic nanoparticles, Phys. Rev. B 65, 144416 (2002).

[10] C. T. Meneses, J. G. S. Duque, E. De Biasi, W. C. Nunes, S. K. Sharma, and M. Knobel, Competing interparticle interactions and surface anisotropy in $\mathrm{NiO}$ nanoparticles, J. Appl. Phys. 108, 013909 (2010).

[11] B. Halamoda-Kenzaoui, M. Ceridono, P. Urbán, A. Bogni, J. Ponti, S. Gioria, and A. Kinsner-Ovaskainen, The agglomeration state of nanoparticles can influence the mechanism of their cellular internalisation, J. Nanobiotechnol. 15, 48 (2017).

[12] B. Mehdaoui, R. P. Tan, A. Meffre, J. Carrey, S. Lachaize, B. Chaudret, and M. Respaud, Increase of magnetic hyperthermia efficiency due to dipolar interactions in lowanisotropy magnetic nanoparticles: Theoretical and experimental results, Phys. Rev. B 87, 174419 (2013).

[13] E. Lima, Jr., E. De Biasi, M. Vasquez Mansilla, M. E. Saleta, M. Granada, H. E. Troiani, F. B. Effenberger, L. M. Rossi, H. R. Rechenberg, and R. D. Zysler, Heat generation in agglomerated ferrite nanoparticles in an alternating magnetic field, J. Phys. D 46, 045002 (2012).

[14] M. Palihawadana-Arachchige, H. Nemala, V. M. Naik, and R. Naik, Effect of magnetic dipolar interactions on temperature dependent magnetic hyperthermia in ferrofluids, J. Appl. Phys. 121, 023901 (2017).

[15] F. H. Sánchez, P. Mendoza Zélis, M. L. Arciniegas, G. A. Pasquevich, and M. B. Fernández van Raap, Dipolar interaction and demagnetizing effects in magnetic nanoparticle dispersions: Introducing the mean-field interacting superparamagnet model, Phys. Rev. B 95, 134421 (2017).

[16] O. Laslett, S. Ruta, J. Barker, R. W. Chantrell, G. Friedman, and O. Hovorka, Interaction effects enhancing magnetic particle detection based on magneto-relaxometry, Appl. Phys. Lett. 106, 012407 (2015).

[17] D. F. Coral, P. Mendoza Zélis, M. Marciello, M. d. P. Morales, A. Craievich, F. H. Sánchez, and M. B. Fernández van Raap, Effect of nanoclustering and dipolar interactions in heat generation for magnetic hyperthermia, Langmuir 32, 1201 (2016).

[18] P. Hugounenq, M. Levy, D. Alloyeau, L. Lartigue, E. Dubois, V. Cabuil, C. Ricolleau, S. Roux, C. Wilhelm,
F. Gazeau, and R. Bazzi, Iron oxide monocrystalline nanoflowers for highly efficient magnetic hyperthermia, J. Phys. Chem. C 116, 15702 (2012).

[19] S. Behrens, W. Habicht, K. Wagner, and E. Unger, Assembly of nanoparticle ring structures based on protein templates, Adv. Mater. 18, 284 (2006).

[20] E. Myrovali, N. Maniotis, A. Makridis, A. Terzopoulou, V. Ntomprougkidis, K. Simeonidis, D. Sakellari, O. Kalogirou, T. Samaras, R. Salikhov, M. Spasova, M. Farle, U. Wiedwald, and M. Angelakeris, Arrangement at the nanoscale: Effect on magnetic particle hyperthermia, Sci. Rep. 6, 37934 (2016).

[21] I. Morales, R. Costo, N. Mille, G. B. Da Silva, J. Carrey, A. Hernando, and P. De la Presa, High frequency hysteresis losses on $\gamma-\mathrm{Fe}_{2} \mathrm{O}_{3}$ and $\mathrm{Fe}_{3} \mathrm{O}_{4}$ : Susceptibility as a magnetic stamp for chain formation, Nanomaterials 8, 970 (2018).

[22] I. Orue, L. Marcano, P. Bender, A. García-Prieto, S. Valencia, M. A. Mawass, D. Gil-Cartón, D. Alba Venero, D. Honecker, A. García-Arribas, L. Fernández Barquín, A. Muela, and M. L. Fdez-Gubieda, Configuration of the magnetosome chain: A natural magnetic nanoarchitecture, Nanoscale 10, 7407 (2018).

[23] C. Martínez-Boubeta, K. Simeonidis, A. Makridis, M. Angelakeris, O. Iglesias, P. Guardia, A. Cabot, L. Yedra, S. Estradé, F. Peiró, Z. Saghi, P. Midgley, I. CondeLeborán, D. Serantes, and D. Baldomir, Learning from nature to improve the heat generation of iron-oxide nanoparticles for magnetic hyperthermia applications, Sci. Rep. 3, 1652 (2013).

[24] D. Serantes, K. Simeonidis, M. Angelakeris, O. ChubykaloFesenko, M. Marciello, M. d. P. Morales, D. Baldomir, and C. Martínez-Boubeta, Multiplying magnetic hyperthermia response by nanoparticle assembling, J. Phys. Chem. C 118, 5927 (2014).

[25] S. Ghaisari, M. Winklhofer, P. Strauch, S. Klumpp, and D. Faivre, Magnetosome organization in magnetotactic bacteria unraveled by ferromagnetic resonance spectroscopy, Biophys. J. 113, 637 (2017).

[26] M. Anand, V. Banerjee, and J. Carrey, Relaxation in onedimensional chains of interacting magnetic nanoparticles: Analytical formula and kinetic Monte Carlo simulations, Phys. Rev. B 99, 024402 (2019).

[27] A. Y. Zubarev, Effect of internal chain-like structures on magnetic hyperthermia in non-liquid media, Phil. Trans. R. Soc. A 377, 20180213 (2019).

[28] A. F. Abu-Bakr and A. Zubarev, Effect of interparticle interaction on magnetic hyperthermia: Homogeneous spatial distribution of the particles, Phil. Trans. R. Soc. A 377, 20180216 (2019).

[29] C. R. Vestal, Q. Song, and Z. J. Zhang, Effects of interparticle interactions upon the magnetic properties of $\mathrm{CoFe}_{2} \mathrm{O}_{4}$ and $\mathrm{MnFe}_{2} \mathrm{O}_{4}$ nanocrystals, J. Phys. Chem. B 108, 18222 (2004).

[30] R. E. Rosensweig, Heating magnetic fluid with alternating magnetic field, J. Magn. Magn. Mater. 252, 370 (2002).

[31] E. De Biasi, R. D. Zysler, C. A. Ramos, and M. Knobel, A new model to describe the crossover from superparamagnetic to blocked magnetic nanoparticles, J. Magn. Magn. Mater. 320, e312 (2008). 
[32] E. De Biasi, E. Lima, Jr., C. A. Ramos, A. Butera, and R. D. Zysler, Effect of thermal fluctuations in FMR experiments in uniaxial magnetic nanoparticles: Blocked vs. superparamagnetic regimes, J. Magn. Magn. Mater. 326, 138 (2013).

[33] E. De Biasi, J. Curiale, and R. D. Zysler, Quantitative study of FORC diagrams in thermally corrected Stoner-Wohlfarth nanoparticles systems, J. Magn. Magn. Mater. 419, 580 (2016).

[34] B. Sanz, M. P. Calatayud, E. De Biasi, E. Lima, Jr., M. Vasquez Mansilla, R. D. Zysler, M. R. Ibarra, and G. F. Goya, In Silico before in vivo: How to predict the heating efficiency of magnetic nanoparticles within the intracellular space, Sci. Rep. 6, 38733 (2016).

[35] E. C. Stoner and E. P. Wohlfarth, A mechanism of magnetic hysteresis in heterogeneous alloys, Phil. Trans. R. Soc. A 240, 599 (1948).

[36] D. Niculaes, A. Lak, G. C. Anyfantis, S. Marras, O. Laslett, S. K. Avugadda, M. Cassani, D. Serantes, O. Hovorka, R. Chantrell, and T. Pellegrino, Asymmetric assembling of iron oxide nanocubes for improving magnetic hyperthermia performance, ACS Nano 11, 12121 (2017).

[37] R. P. Tan, J. Carrey, and M. Respaud, Magnetic hyperthermia properties of nanoparticles inside lysosomes using kinetic Monte Carlo simulations: Influence of key parameters and dipolar interactions, and evidence for strong spatial variation of heating power, Phys. Rev. B 90, 214421 (2014).
[38] J. A. De Toro, P. S. Normile, S. S. Lee, D. Salazar, J. L. Cheong, P. Muñiz, J. M. Riveiro, M. Hillenkamp, F. Tournus, A. Tamion, and P. Nordblad, Controlled close-packing of ferrimagnetic nanoparticles: An assessment of the role of interparticle superexchange versus dipolar interactions, J. Phys. Chem. C 117, 10213 (2013).

[39] J. A. De Toro, S. S. Lee, D. Salazar, J. L. Cheong, P. S. Normile, P. Muñiz, J. M. Riveiro, M. Hillenkamp, F. Tournus, A. Tamion, and P. Nordblad, A nanoparticle replica of the spin-glass state, Appl. Phys. Lett. 102, 183104 (2013).

[40] P. Nordblad, Competing interaction in magnets: The root of ordered disorder or only frustration?, Phys. Scr. 88, 058301 (2013).

[41] D. Cao, H. Li, L. Pan, J. Li, X. Wang, P. Jing, X. Cheng, W. Wang, J. Wang, and Q. Liu, High saturation magnetization of $\gamma-\mathrm{Fe}_{2} \mathrm{O}_{3}$ nano-particles by a facile one-step synthesis approach, Sci. Rep. 6, 32360 (2016).

[42] S. Dutz and R. Hergt, Magnetic nanoparticle heating and heat transfer on a microscale: Basic principles, realities and physical limitations of hyperthermia for tumour therapy, Int. J. Hyperther. 29, 790 (2013).

[43] W. J. Atkinson, I. A. Brezovich, and D. P. Chakraborty, Usable frequencies in hyperthermia with thermal seeds, IEEE Trans. Biomed. Eng. 31, 70 (1984).

[44] A. Stancu and I. Chiorescu, Crossover condition in the coherent rotation model and the Preisach-type models, IEEE Trans. Magn. 33, 2573 (1997). 